\title{
MULTI-DOMAIN SPECTRAL COLLOCATION METHOD FOR VARIABLE-ORDER NONLINEAR FRACTIONAL DIFFERENTIAL EQUATIONS*
}

\author{
TINGGANG ZHAO ${ }^{\dagger}$, ZHIPING MAO $^{\ddagger}$, AND GEORGE EM KARNIADAKIS
}

\begin{abstract}
Spectral and spectral element methods using Galerkin type formulations are efficient for solving linear fractional PDEs (FPDEs) of constant order but are not efficient in solving nonlinear FPDEs and cannot handle FPDEs with variable-order. In this paper, we present a multidomain spectral collocation method that addresses these limitations. We consider FPDEs in the Riemann-Liouville sense, and employ Jacobi Lagrangian interpolants to represent the solution in each element. We provide variable-order differentiation formulas, which can be computed efficiently for the multi-domain discretization taking into account the nonlocal interactions. We enforce the interface continuity conditions by matching the solution values at the element boundaries via the Lagrangian interpolants, and in addition we minimize the jump in (integer) fluxes using a penalty method. We analyze numerically the effect of the penalty parameter on the condition number of the global differentiation matrix and on the stability and convergence of the penalty collocation scheme. We demonstrate the effectiveness of the new method for the fractional Helmholtz equation of constant and variable-order using $h-p$ refinement for different values of the penalty parameter. We also solve the fractional Burgers equation with constant and variable-order and compare with solutions obtained with a single domain spectral collocation method.
\end{abstract}

Key words. Penalty method, $h-p$ refinement, Fractional Helmholtz equation, Fractional Burgers equation

AMS subject classifications. 65N35, 65M70, 41A05, 41A25

1. Introduction. Recently, fractional differential equations (FDEs) have been applied in modeling a wide range of complex physical processes, for example, anomalous transport processes [4, 27, 26], frequency-dependent damping behavior in viscoelastic materials [8, 1, 2], continuum and statistical mechanics [19, solid mechanic 28, economics [3], and so on. Moreover, FDEs with variable-order can more accurately describe the anomalous diffusion since the diffusion rate may be time or space dependent, see [12, 29] and references therein.

Close forms of solutions of even linear FDEs, especially those with variable-order, is difficult to obtain. Thus, robust numerical methods are required to discretize the fractional operators. Proposed numerical methods so far include extensions of the finite difference method (FDM) [17, 18, 24, 25, 31, 33, 32, 35, 36, 46, 49, 47 as well as of the finite element method (FEM) [7, 9, 10, 16, 37, 48, and references therein. However, since the fractional operators are non-local, then both FDM and FEM, which are local methods, would lose a big advantage that they enjoy for classical PDEs with locally defined derivatives. In contrast, global methods, such as the spectral method, could play an important role in developing efficient and highly accurate numerical discretizations for FDEs, see [13, 14, 15, 34, 38, 20, 22, 43, 42] and references therein. It is well-known that the solutions of FDEs exhibit end-point singularities even with

\footnotetext{
†School of Mathematics, Lanzhou City University, Lanzhou 730070, China (tinggang_zhao@brown.edu).

${ }^{\ddagger}$ Division of Applied Mathematics, Brown University, Providence, RI 02912, USA (zhiping_mao@brown.edu, george_karniadakis@brown.edu).

$\S$ Corresponding author.

* This work was supported by the MURI/ARO on "Fractional PDEs for Conservation Laws and Beyond: Theory, Numerics and Applications" (W911NF-15-1-0562). The research of the first author was partially supported by NSF of China (No. 11661048).
} 
smooth source terms. This causes additional difficulties in developing high accuracy methods for FDEs. To resolve this issue, an efficient and highly accurate spectral method was proposed in [39] by using poly-fractonomials, which are eigenfunctions of a fractional Sturm-Liouville operator, as basis functions, leading to sparse matrices for simple model equations (linear equation without reaction term) of constant order; a rigorous error analysis was established in [6] showing that the error only depends on the regularity of the right-hand function. This idea was also extended to Riesz FDEs [20] and general two-sided FDEs [21. However, such approach may fail for solving more complex FDEs, such as FDEs with a reaction term, variable-order FDEs or nonlinear problems, due to the fact that the singular behavior of the end points is hard to match with one single set of basis functions.

To this end, a more flexible method, which combines domain decomposition with spectral expansions, namely the spectral element method (SEM, also called multidomain spectral method), was introduced. For instance, Mao and Shen constructed a high accuracy SEM based on the geometric mesh showing that the error has exponential decay with respect to the square root of the number of degrees of freedom without prior knowledge about the singular behavior [23. Some other SEMs can be found in [40, 41, 11. However, the aforementioned SEMs are based on Galerkin or Petrov-Galerkin type formulations, which are not efficient in dealing with nonlinear problems. Moreover, they cannot handle FDEs of variable-order. In contrast, the collocation type method does not suffer from such issues, and is particularly suitable for the variable-order fractional problem. In [45, 44], the authors developed a generalized spectral collocation method with tunable accuracy for FDEs of variable-order by using the weighted Jacobi polynomials. Although effective, the collocation method relies on empirically tuning the basis functions to capture the singular terms and cannot target special discretization strategies, e.g. exploiting the strength of graded or geometric meshes.

In this paper, we aim to develop a stable and highly accurate multi-domain spectral collocation method (MDSCM) for solving variable-order nonlinear FDEs in the Riemann-Liouville sense. In particular, we employ Jacobi Lagrangian interpolants to represent the solution in each element. Directly applying the MDSCM to solve FDEs may lead to instabilities. Thus, we employ a penalty technique at the element interfaces by minimizing the jump in (integer) fluxes to stabilize the MDSCM. The penalty technique was also used previously in 38 for FDEs in the Caputo sense. The main contributions of this work are as follows:

- We first construct a set of $C^{0}$ nodal basis functions and derive the corresponding variable-order differentiation matrix for the multi-domain discretization taking into account the nonlocal interactions. We also provide an efficient algorithm to compute the entries of the differentiation matrix by hybridizing the three-term recurrence relation and the Gauss quadrature.

- We enforce the interface continuity conditions by matching the solution values at the element boundaries via the Lagrangian interpolants, and in addition we minimize the jump in (integer) fluxes using a penalty method. We also analyze numerically the effect of the penalty parameter on the condition number of the global differentiation matrix and the stability of the discretized scheme.

- We demonstrate the effectiveness of the new method for the fractional Helmholtz equation of constant and variable-order using $h-p$ refinement for different values of the penalty parameter, and solve the fractional Burgers equation with constant and variable order, and compare with solutions obtained using 
a single-domain spectral collocation method.

The paper is organized as follows. In the next section, we present some definitions and properties for variable-order fractional integrals and derivatives. We propose the MDSCM in detail in section 3, Moreover, in subsection 3.3. we discuss the eigenvalues of the multi-domain fractional differentiation matrix and introduce the penalty technique to stabilize the MDSCM. We present several numerical examples for the fractional Helmholtz equation and the fractional Burgers equation in section 4 Finally, we conclude in section 5 .

2. Preliminaries. In this section, we review definitions of variable-order fractional integrals and derivatives $([45,50])$.

DeFinition 2.1. For $\rho(x)>0$, the left and right fractional integrals in the sense of Riemann-Liouville are defined as

$$
{ }_{a} I_{x}^{\rho(x)} v(x)=\frac{1}{\Gamma(\rho(x))} \int_{a}^{x} \frac{v(y)}{(x-y)^{1-\rho(x)}} d y, \quad x \in[a, b],
$$

and

$$
{ }_{x} I_{b}^{\rho(x)} v(x)=\frac{1}{\Gamma(\rho(x))} \int_{x}^{b} \frac{v(y)}{(y-x)^{1-\rho(x)}} d y, \quad x \in[a, b],
$$

respectively, where $\Gamma(\cdot)$ is the Euler's Gamma function.

Definition 2.2. For $k-1<\rho(x)<k$ with $k \in \mathbb{N}$, the left and right RiemannLiouville derivative of order $\rho(x)$ are defined as

$$
{ }_{a} D_{x}^{\rho(x)} v(x)=\frac{1}{\Gamma(k-\rho(x))}\left[\frac{d^{k}}{d \xi^{k}} \int_{a}^{\xi} \frac{v(y)}{(\xi-y)^{\rho(x)-k+1}} d y\right]_{\xi=x},
$$

and

$$
{ }_{x} D_{b}^{\rho}(x) v(x)=\frac{(-1)^{k}}{\Gamma(k-\rho(x))}\left[\frac{d^{k}}{d \xi^{k}} \int_{\xi}^{b} \frac{v(y)}{(y-\xi)^{\rho(x)-k+1}} d y\right]_{\xi=x}
$$

for $x \in[a, b]$, respectively.

Similar to the constant-order case, there exist some well-known properties for the variable-order case, for instance, if $n>\rho(x)$ or $n-\rho(x) \notin \mathbb{Z}$,

$$
{ }_{a} D_{x}^{\rho(x)}(x-a)^{n}=\frac{\Gamma(n+1)}{\Gamma(n+1-\rho(x))}(x-a)^{n-\rho(x)} .
$$

For $k-1<\alpha<k$ with $k \in \mathbb{N}$, let us introduce the following notation:

$$
\widetilde{D}_{a}^{b, \rho(x)} u(x)=\frac{1}{\Gamma(k-\rho(x))}\left[\frac{d^{k}}{d \xi^{k}} \int_{a}^{b} \frac{u(y)}{(\xi-y)^{\rho(x)-k+1}} d y\right]_{\xi=x}, \quad x>b .
$$

It can be verified that

$$
{ }_{a} D_{x}^{\rho(x)} u(x)={ }_{b} D_{x}^{\rho(x)} u(x)+\widetilde{D}_{a}^{b, \rho(x)} u(x), \quad a<b<x .
$$

3. Multi-domain fractional differentiation matrix of variable-order. We introduce in this section the multi-domain fractional differentiation matrix (MDFDM) of variable-order and provide an efficient algorithm to compute its entries. We also present how to minimize the jump in the (integer) fluxes using a penalty method. 


\subsection{Multi-domain fractional differentiation matrix of variable-order.} Let $\Lambda:=\left(x_{L}, x_{R}\right)$, we first divide the interval $\Lambda$ into $M$ elements, i.e.,

$$
x_{L}=x_{0}<x_{1}<\cdots<x_{M}=x_{R} .
$$

Denote $I_{k}=\left[x_{k-1}, x_{k}\right], k=1, \ldots, M$ the $k$-th element and $h_{k}=x_{k}-x_{k-1}$ the length of $I_{k}$. Let $\mathbb{P}_{N}^{I}$ be the collection of all algebraic polynomials defined on interval $I$ with degree at most $N$. We now introduce the piecewise polynomial space

$$
\mathbb{V}_{N}=\left\{v \in C(\Lambda):\left.v\right|_{I_{k}} \in \mathbb{P}_{N_{k}}^{I_{k}}\right\}
$$

where $N_{k}, k=1, \ldots, M$ are all positive integers. For each $k=1,2, \ldots, M$, we select a set of collocation points in $I_{k}$, denoted by $\left\{x_{j}^{k}\right\}_{j=0}^{N_{k}}$, satisfying $x_{0}^{k}=x_{k-1}$ and $x_{N_{k}}^{k}=x_{k}$. We collect all these points and denote

$$
\mathbb{N}_{o}:=\left\{x_{j}^{k}: k=1, \ldots, M ; j=0, \ldots, N_{k}\right\} .
$$

The magnitude of $\mathbb{N}_{o}$ is $\sum_{k=1}^{M} N_{k}+1$.

For $k=1,2, \ldots, M$ and $j=0, \ldots, N_{k}$, we denote by $L_{j, k}(x)$ the $j$-th Lagrange interpolation polynomial on element $I_{k}$ satisfying $L_{j, k}\left(x_{i}^{k}\right)=\delta_{i j}$. Let us first define a set of basis functions. For the boundary points and interface points, the corresponding basis functions are given by

$$
\phi_{0}(x)=\left\{\begin{array}{ll}
L_{0,1}(x), & \text { if } x \in I_{1}, \\
0, & \text { otherwise, }
\end{array} \quad \phi_{M}(x)= \begin{cases}L_{N_{M}, M}(x), & \text { if } x \in I_{M}, \\
0, & \text { otherwise }\end{cases}\right.
$$

and

$$
\phi_{k}(x)= \begin{cases}L_{N_{k}, k}(x), & \text { if } x \in I_{k}, \\ L_{0, k+1}(x), & \text { if } x \in I_{k+1}, \\ 0, & \text { otherwise, }\end{cases}
$$

$k=1, \ldots, M-1$, respectively, while for the interior points of each element, the basis functions are given by

$$
\psi_{j}^{k}(x)=\left\{\begin{array}{ll}
L_{j, k}(x), & \text { if } x \in I_{k}, \\
0, & \text { otherwise, }
\end{array} \quad k=1, \ldots, M, j=1, \ldots, N_{k}-1 .\right.
$$

Therefore, we have

$$
\mathbb{V}_{N}=\operatorname{span}\left\{\phi_{k}, k=0, \ldots, M\right\} \cup \operatorname{span}\left\{\psi_{j}^{k}, k=1, \ldots, M ; j=1, \ldots, N_{k}-1\right\} .
$$

For $u_{N} \in \mathbb{V}_{N}$, it can be expanded as

$$
u_{N}(x)=\sum_{k=1}^{M} \sum_{j=1}^{N_{k}-1} u_{N}\left(x_{j}^{k}\right) \psi_{j}^{k}(x)+\sum_{k=0}^{M} u_{N}\left(x_{k}\right) \phi_{k}(x) .
$$

Taking the fractional derivative of order $\alpha(x)$ and evaluating the values at all collocation points, we obtain the MDFDM of $\alpha(x)$, denoted by $\mathbf{D}^{\alpha}$, as follows

$$
\mathbf{D}^{\alpha}=\left[\begin{array}{ccccc}
\widehat{\mathbf{D}}^{11} & & & & \overline{\mathbf{D}}^{1} \\
\widehat{\mathbf{D}}^{21} & \widehat{\mathbf{D}}^{22} & & & \overline{\mathbf{D}}^{2} \\
\vdots & \vdots & \ddots & & \vdots \\
\widehat{\mathbf{D}}^{M 1} & \widehat{\mathbf{D}}^{M 2} & \cdots & \widehat{\mathbf{D}}^{M M} & \overline{\mathbf{D}}^{M} \\
\underline{\mathbf{D}}^{1} & \underline{\mathbf{D}}^{2} & \cdots & \underline{\mathbf{D}}^{M} & \widetilde{\mathbf{D}}
\end{array}\right]
$$


where $\widehat{\mathbf{D}}^{i j}$ denotes the differentiation matrix associated with the inner points of the $i$-th and $j$-th elements given by

$$
\widehat{\mathbf{D}}^{i j}=\left[{ }_{x_{L}} D_{x}^{\alpha\left(x_{m}^{j}\right)} \psi_{n}^{i}\left(x_{m}^{j}\right)\right]_{m, n=1, \ldots, N_{i}-1}, i, j=1, \ldots, M .
$$

$\overline{\mathbf{D}}^{i}$ and $\underline{\mathbf{D}}^{i}$ denote the differentiation matrices associated with the inner points of the $i$-th element and interface points given by

$$
\overline{\mathbf{D}}^{i}=\left[{ }_{x_{L}} D_{x}^{\alpha\left(x_{m}^{i}\right)} \phi_{n}\left(x_{m}^{i}\right)\right]_{m=1, \ldots, N_{i}-1 ; n=1, \ldots, M-1}
$$

and

$$
\underline{\mathbf{D}}^{i}=\left[{ }_{x_{L}} D_{x}^{\alpha\left(x_{m}\right)} \psi_{n}^{i}\left(x_{m}\right)\right]_{m=1, \ldots, M-1 ; n=1, \ldots, N_{i}-1}, i=1, \ldots, M
$$

respectively, and $\widetilde{\mathbf{D}}$ denotes the differentiation matrix associated with the interface points given by

$$
\widetilde{\mathbf{D}}=\left[{ }_{x_{L}} D_{x}^{\alpha\left(x_{m}\right)} \phi_{n}\left(x_{m}\right)\right]_{m, n=1, \ldots, M-1} .
$$

We show the structure of the differentiation matrix $\mathbf{D}^{\alpha}$ with $M=5, N_{i}=4, i=$ $1, \ldots, 5$ in Fig. 3.1 the entries of the white blocks are zeros while the entries of colored blocks are nonzero. The entries with different colors are evaluated by different formulas proposed in the next subsection. All the nodal basis functions defined above are

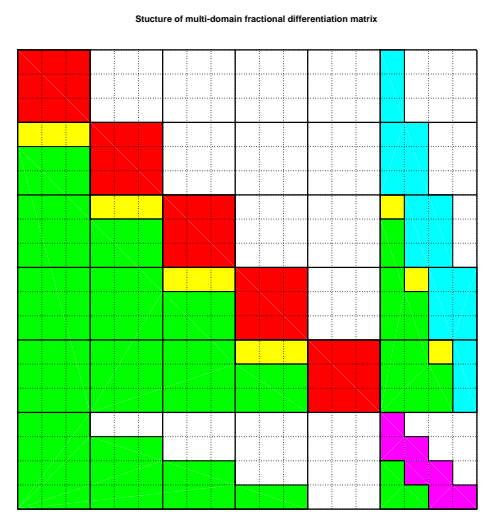

Fig. 3.1: Structure of multi-domain fractional differentiation matrix with $M=5, N_{i}=$ $4, i=1, \ldots, 5$.

$C\left[x_{L}, x_{R}\right]$. The following result shows that $\lim _{x \rightarrow x_{i}+} x_{L} D_{x}^{\alpha} \phi_{i}(x)=\infty, i=1, \ldots, M-1$ even for a constant $\alpha>1$. In our case, we only use values of the fractional derivatives of basis functions from left at the interface points, i.e., $\lim _{x \rightarrow x_{i}-} x_{L} D_{x}^{\alpha} \phi_{i}(x), i=$ $1, \ldots, M-1$, when $\alpha>1$. 
Lemma 3.1. Let $\alpha \in(0,2)$ be a constant, $a<c<b$. If $u \in C^{2}[a, c] \cap C^{2}[c, b] \cap$ $C[a, b]$ and $u^{\prime}\left(c^{-}\right)$and $u^{\prime}\left(c^{+}\right)$exist, then we have

$$
\begin{aligned}
{ }_{a} D_{x}^{\alpha} u(x)= & \frac{(x-a)^{-\alpha}}{\Gamma(1-\alpha)} u(a)+\frac{(x-a)^{1-\alpha}}{\Gamma(2-\alpha)} u^{\prime}(a) \\
& +\frac{(x-c)^{1-\alpha}}{\Gamma(2-\alpha)}\left[u^{\prime}\left(c^{+}\right)-u^{\prime}\left(c^{-}\right)\right]+s(x),
\end{aligned}
$$

for any $x \in(c, b)$, where

$$
s(x)= \begin{cases}\frac{1}{\Gamma(3-\alpha)} \frac{d}{d x}\left[\int_{a}^{x} u^{\prime \prime}(\tau)(x-\tau)^{2-\alpha} d \tau\right], & \text { if } \alpha \in(0,1), \\ \frac{1}{\Gamma(4-\alpha)} \frac{d^{2}}{d x^{2}}\left[\int_{a}^{x} u^{\prime \prime}(\tau)(x-\tau)^{3-\alpha} d \tau\right], & \text { if } \alpha \in(1,2) .\end{cases}
$$

Proof. For $\alpha \in(0,1)$, using the integration by parts, we have

$$
\begin{aligned}
{ }_{a} D_{x}^{\alpha} u(x)= & \frac{1}{\Gamma(1-\alpha)} \frac{d}{d x}\left[\int_{a}^{c}(x-\tau)^{-\alpha} u(\tau) d \tau+\int_{c}^{x}(x-\tau)^{-\alpha} u(\tau) d \tau\right] \\
= & \frac{1}{\Gamma(1-\alpha)} \frac{d}{d x}\left[\frac{(x-a)^{1-\alpha} u(a)-(x-c)^{1-\alpha} u\left(c^{-}\right)}{1-\alpha}\right. \\
& +\frac{(x-a)^{2-\alpha} u^{\prime}(a)-(x-c)^{2-\alpha} u^{\prime}\left(c^{-}\right)}{(1-\alpha)(2-\alpha)}+\frac{\int_{a}^{c}(x-\tau)^{2-\alpha} u^{\prime \prime}(\tau) d \tau}{(1-\alpha)(2-\alpha)} \\
& \left.+\frac{(x-c)^{1-\alpha} u\left(c^{+}\right)}{1-\alpha}+\frac{(x-c)^{2-\alpha} u^{\prime}\left(c^{+}\right)}{(1-\alpha)(2-\alpha)}+\frac{\int_{a}^{c}(x-\tau)^{2-\alpha} u^{\prime \prime}(\tau) d \tau}{(1-\alpha)(2-\alpha)}\right] .
\end{aligned}
$$

Then, the result follows from $u\left(c^{-}\right)=u\left(c^{+}\right)$for $\alpha \in(0,1)$. For $\alpha \in(1,2)$, we obtain the result by using a similar argument. $\square$

3.2. Computing the differentiation matrix. We now present how to compute the entries of the MDFDM, i.e., how to perform the computation of ${ }_{x_{L}} D_{x}^{\alpha(x)} \phi_{k}(x)$, $k=0,1, \ldots, M$ and ${ }_{x_{L}} D_{x}^{\alpha(x)} \psi_{j}^{k}(x)$ for $k=1,2, \ldots, M, j=1,2, \ldots, N_{k}-1, x \in \mathbb{N}_{o}$.

By the definition of nodal basis functions, we have for $k=1, \ldots, M-1$,

$$
{ }_{x_{L}} D_{x}^{\alpha(x)} \phi_{k}(x)= \begin{cases}0, & \text { if } x \leq x_{k-1}, \\ x_{k-1} D_{x}^{\alpha(x)} L_{N_{k}, k}(x), & \text { if } x_{k-1}<x \leq x_{k}, \\ \widetilde{D}_{x_{k}, \alpha-1}^{x_{k}(x)} L_{N_{k}, k}(x)+x_{k} D_{x}^{\alpha(x)} L_{0, k+1}(x), & \text { if } x_{k}<x \leq x_{k+1}, \\ \widetilde{D}_{x_{k-1}, \alpha(x)}^{x_{k}, \alpha} L_{N_{k}, k}(x)+\widetilde{D}_{x_{k}}^{x_{k+1, \alpha(x)}} L_{0, k+1}(x), & \text { if } x>x_{k+1},\end{cases}
$$

and

$$
\begin{aligned}
x_{L} D_{x}^{\alpha(x)} \phi_{0}(x) & = \begin{cases}x_{0} D_{x}^{\alpha(x)} L_{0,1}(x), & \text { if } x_{0}<x \leq x_{1}, \\
\widetilde{D}_{x_{0}}^{x_{1}, \alpha(x)} L_{0,1}(x), & \text { if } x>x_{1},\end{cases} \\
x_{L} D_{x}^{\alpha(x)} \phi_{M}(x) & = \begin{cases}0, & \text { if } x \leq x_{M-1}, \\
x_{M-1} D_{x}^{\alpha(x)} L_{N_{M}, M}(x), & \text { if } x_{M-1}<x \leq x_{M} .\end{cases}
\end{aligned}
$$

Moreover, for $k=1, \ldots, M$ and $j=1, \ldots, N_{k}$, we have

$$
x_{L} D_{x}^{\alpha(x)} \psi_{j}^{k}(x)= \begin{cases}0, & \text { if } x \leq x_{k-1}, \\ x_{k-1} D_{x}^{\alpha(x)} L_{j, k}(x), & \text { if } x_{k-1}<x \leq x_{k}, \\ \widetilde{D}_{x_{k-1}}^{x_{k}, \alpha(x)} L_{j, k}(x), & \text { if } x>x_{k} .\end{cases}
$$


Overall, we observe that we shall compute the following two types of integral:

$$
x_{k-1} D_{x}^{\alpha(x)} L_{j, k}(x), x \in\left(x_{k-1}, x_{k}\right] \quad \text { and } \quad \widetilde{D}_{x_{k-1}}^{x_{k}, \alpha(x)} L_{j, k}(x), \quad x>x_{k} .
$$

By using the transformation $x=\frac{h_{k}}{2}(y+1)+x_{k-1} \in I_{k}$, we arrive at

$$
x_{k-1} D_{x}^{\alpha(x)} L_{j, k}(x)=\left(\frac{2}{h_{k}}\right)^{\hat{\alpha}(y)}{ }_{-1} D_{y}^{\hat{\alpha}(y)} L_{j, k}(y)
$$

and

$$
\widetilde{D}_{x_{k-1}}^{x_{k}, \alpha(x)} L_{j, k}(x)=\left(\frac{2}{h_{k}}\right)^{\hat{\alpha}(y)} \widetilde{D}_{-1}^{1, \hat{\alpha}(y)} L_{j, k}(y),
$$

For the sake of simplicity, we drop the index $k$ and let $L_{j}(y)$ be the $j$-th Lagrange interpolation polynomial associated with nodes $\left\{y_{i}\right\}_{i=0}^{N} \in[-1,1]$. The following results play an important role in efficiently computing the above two types of integral. The first one can be obtained by replacing $\alpha$ with $k-\alpha$ in the equation (3.9) of [13].

Theorem 3.2. For $k-1<\alpha(y)<k, k \in \mathbb{N}$, let

$$
\hat{R}_{j}^{c, d, \alpha(y)}(y):=\frac{1}{\Gamma(k-\alpha(y))} \int_{-1}^{y} \frac{P_{j}^{c, d}(s)}{(y-s)^{\alpha(y)-k+1}} d s, \quad y \in[-1,1] .
$$

Then, $\left\{\hat{R}_{j}^{c, d, \alpha(y)}(y)\right\}_{j=0}^{N}$ satisfies the following three-term recurrence relation:

$$
\begin{aligned}
\hat{R}_{0}^{c, d, \alpha(y)}(y)= & \frac{(y+1)^{k-\alpha(y)}}{\Gamma(k-\alpha(y)+1)}, \\
\hat{R}_{1}^{c, d, \alpha(y)}(y)= & P_{1}^{c, d}(-1) \frac{(y+1)^{k-\alpha(y)}}{\Gamma(k-\alpha(y)+1)}+\frac{c+d+2}{2} \frac{(y+1)^{k-\alpha(y)+1}}{\Gamma(k-\alpha(y)+2)}, \\
\hat{R}_{j+1}^{c, d, \alpha(y)}(y)= & \left(\widetilde{A}_{j}^{c, d, \alpha(y)} y-\widetilde{B}_{j}^{c, d, \alpha(y)}\right) \hat{R}_{j}^{c, d, \alpha(y)}(y) \\
& -\widetilde{C}_{j}^{c, d, \alpha(y)} \hat{R}_{j-1}^{c, d, \alpha(y)}(y)+\frac{\widetilde{D}_{j}^{c, d, \alpha(y)}}{\Gamma(k-\alpha(y))}(y+1)^{k-\alpha(y)},
\end{aligned}
$$

for $j \geq 1$, where

$$
\begin{aligned}
\widetilde{A}_{j}^{c, d, \alpha(y)} & =\frac{A_{j}^{c, d}}{1+(k-\alpha(y)) A_{j}^{c, d} \widehat{C}_{j}^{c, d}}, \\
\widetilde{B}_{j}^{c, d, \alpha(y)} & =\frac{B_{j}^{c, d}+(k-\alpha(y)) A_{j}^{c, d} \widehat{B}_{j}^{c, d}}{1+(k-\alpha(y)) A_{j}^{c, d} \widehat{C}_{j}^{c, d}} \\
\widetilde{C}_{j}^{c, d, \alpha(y)} & =\frac{C_{j}^{c, d}+(k-\alpha(y)) A_{j}^{c, d} \widehat{A}_{j}^{c, d}}{1+(k-\alpha(y)) A_{j}^{c, d} \widehat{C}_{j}^{c, d}} \\
\widetilde{D}_{j}^{c, d, \alpha(y)} & =\frac{A_{j}^{c, d}\left(\widehat{A}_{j}^{c, d} P_{j-1}^{c, d}(-1)+\widehat{B}_{j}^{c, d} P_{j}^{c, d}(-1)+\widehat{C}_{j}^{c, d} P_{j+1}^{c, d}(-1)\right)}{1+(k-\alpha(y)) A_{j}^{c, d} \widehat{C}_{j}^{c, d}},
\end{aligned}
$$

$A_{j}^{c, d}, B_{j}^{c, d}$ and $C_{j}^{c, d}$ can be found in [30, Equation (3.111)], $\widehat{A}_{j}^{c, d}, \widehat{B}_{j}^{c, d}$ and $\widehat{C}_{j}^{c, d}$ can be found in [30, Equation (3.124)]. 
The next theorem is an extension of [5, Theorem 3.1] for the variable-order case.

Theorem 3.3. For $k-1<\alpha(y)<k$ and $k \in \mathbb{N}$, let

$$
\breve{R}_{j}^{c, d, \alpha(y)}(y):=\frac{1}{\Gamma(k-\alpha(y))} \int_{-1}^{1} \frac{P_{j}^{c, d}(s)}{(y-s)^{\alpha(y)-k+1}} d s, \quad y>1 .
$$

Then, $\left\{\breve{R}_{j}^{c, d, \alpha(y)}(y)\right\}_{j=0}^{N}$ satisfies the following three-term recurrence relation:

$$
\begin{aligned}
\breve{R}_{0}^{c, d, \alpha(y)}(y)= & \frac{(y+1)^{k-\alpha(y)}-(y-1)^{k-\alpha(y)}}{\Gamma(k-\alpha(y)+1)}, \\
\breve{R}_{1}^{c, d, \alpha(y)}(y)= & \frac{P_{1}^{c, d}(-1)(y+1)^{k-\alpha(y)}-P_{1}^{c, d}(1)(y-1)^{k-\alpha(y)}}{\Gamma(k-\alpha(y)+1)} \\
& +\frac{(c+d+2)\left((y+1)^{k-\alpha(y)+1}-(y-1)^{k-\alpha(y)+1}\right)}{2 \Gamma(k-\alpha(y)+2)}, \\
\breve{R}_{j+1}^{c, d, \alpha(y)}(y)= & \left(\widetilde{A}_{j}^{c, d, \alpha(y)} y-\widetilde{B}_{j}^{c, d, \alpha(y)}\right) \breve{R}_{j}^{c, d, \alpha(y)}(y)-\widetilde{C}_{j}^{c, d, \alpha(y)} \breve{R}_{j-1}^{c, d, \alpha(y)}(y) \\
& +\frac{\widetilde{D}_{j}^{c, d, \alpha(y)}}{\Gamma(k-\alpha(y))}(y+1)^{k-\alpha(y)}-\frac{\widetilde{E}_{j}^{c, d, \alpha(y)}}{\Gamma(k-\alpha(y))}(y-1)^{k-\alpha(y)},
\end{aligned}
$$

for $j \geq 1$, where $\widetilde{A}_{j}^{c, d, \alpha(y)}, \widetilde{B}_{j}^{c, d, \alpha(y)}, \widetilde{C}_{j}^{c, d, \alpha(y)}, \widetilde{D}_{j}^{c, d, \alpha(y)}$ are given in (3.5) and

$$
\widetilde{E}_{j}^{c, d, \alpha(y)}=\frac{A_{j}^{c, d}\left(\widehat{A}_{j}^{c, d} P_{j-1}^{c, d}(1)+\widehat{B}_{j}^{c, d} P_{j}^{c, d}(1)+\widehat{C}_{j}^{c, d} P_{j+1}^{c, d}(1)\right)}{1+(k-\alpha(y)) A_{j}^{c, d} \widehat{C}_{j}^{c, d}} .
$$

We next compute the integer derivatives of $\hat{R}_{j}^{c, d, \alpha(y)}(y)$ and $\breve{R}_{j}^{c, d, \alpha(y)}(y)$ up to order $k$. To this end, we have the following results:

TheOREm 3.4. Let $k-1<\alpha(y)<k, 1 \leq m \leq k$ and $k, m \in \mathbb{N}$, we have the following three-term recurrence relation: for $j \geq 1$,

$$
\begin{aligned}
\frac{d^{m}}{d y^{m}} \hat{R}_{0}^{c, d, \alpha(y)}(y)= & \frac{(y+1)^{k-\alpha(y)-m}}{\Gamma(k-\alpha(y)+1-m)}, \\
\frac{d^{m}}{d y^{m}} \hat{R}_{1}^{c, d, \alpha(y)}(y)= & P_{1}^{c, d}(-1) \frac{(y+1)^{k-\alpha(y)-m}}{\Gamma(k-\alpha(y)+1-m)}+\frac{c+d+2}{2} \frac{(y+1)^{k-\alpha(y)+1-m}}{\Gamma(k-\alpha(y)+2-m)}, \\
\frac{d^{m}}{d y^{m}} \hat{R}_{j+1}^{c, d, \alpha(y)}(y)= & \left(\widetilde{A}_{j}^{c, d, \alpha(y)} y-\widetilde{B}_{j}^{c, d, \alpha(y)}\right) \frac{d^{m}}{d y^{m}} \hat{R}_{j}^{c, d, \alpha(y)}(y) \\
& -\widetilde{C}_{j}^{c, d, \alpha(y)} \frac{d^{m}}{d y^{m}} \hat{R}_{j-1}^{c, d, \alpha(y)}(y)+m \widetilde{A}_{j}^{c, d, \alpha(y)} \frac{d^{m-1}}{d y^{m-1}} \hat{R}_{j}^{c, d, \alpha(y)}(y) \\
& +\frac{(k-\alpha(y)) \widetilde{D}_{j}^{c, d, \alpha(y)}}{\Gamma(k-\alpha(y)+1-m)}(y+1)^{k-\alpha(y)-m},
\end{aligned}
$$

where $\widetilde{A}_{j}^{c, d, \alpha(y)}, \widetilde{B}_{j}^{c, d, \alpha(y)}, \widetilde{C}_{j}^{c, d, \alpha(y)}, \widetilde{D}_{j}^{c, d, \alpha(y)}$ are given in (3.5).

Theorem 3.5. Let $k-1<\alpha(y)<k, 1 \leq m \leq k$ and $k, m \in \mathbb{N}$, we have the 
following three-term recurrence relation: for $j \geq 1$,

$$
\begin{aligned}
\frac{d^{m}}{d y^{m}} \breve{R}_{0}^{c, d, \alpha(y)}(y)= & \frac{(y+1)^{k-\alpha(y)-m}-(y-1)^{k-\alpha(y)-m}}{\Gamma(k-\alpha(y)+1-m)}, \\
\frac{d^{m}}{d y^{m}} \breve{R}_{1}^{c, d, \alpha(y)}(y)= & \frac{P_{1}^{c, d}(-1)(y+1)^{k-\alpha(y)-m}-P_{1}^{c, d}(1)(y-1)^{k-\alpha(y)-m}}{\Gamma(k-\alpha(y)+1-m)} \\
& +\frac{(c+d+2)\left((y+1)^{k-\alpha(y)+1-m}-(y-1)^{k-\alpha(y)+1-m}\right)}{2 \Gamma(k-\alpha(y)+2-m)} \\
\frac{d^{m}}{d y^{m}} \breve{R}_{j+1}^{c, d, \alpha(y)}(y)= & \left(\widetilde{A}_{j}^{c, d, \alpha(y)} y-\widetilde{B}_{j}^{c, d, \alpha(y)}\right) \frac{d^{m}}{d y^{m}} \breve{R}_{j}^{c, d, \alpha(y)}(y) \\
& -\widetilde{C}_{j}^{c, d, \alpha(y)} \frac{d^{m}}{d y^{m}} \breve{R}_{j-1}^{c, d, \alpha(y)}(y)+m \widetilde{A}_{j}^{c, d, \alpha(y)} \frac{d^{m-1}}{d y^{m-1}} \breve{R}_{j}^{c, d, \alpha(y)}(y) \\
& +\frac{\widetilde{D}_{j}^{c, d, \alpha(y)}(y+1)^{k-\alpha(y)-m}-\widetilde{E}_{j}^{c, d, \alpha(y)}(y-1)^{k-\alpha(y)-m}}{(k-\alpha(y))^{-1} \Gamma(k-\alpha(y)+1-m)},
\end{aligned}
$$

where $\widetilde{A}_{j}^{c, d, \alpha(y)}, \widetilde{B}_{j}^{c, d, \alpha(y)}, \widetilde{C}_{j}^{c, d, \alpha(y)}, \widetilde{D}_{j}^{c, d, \alpha(y)}$ are given in (3.5) and $\widetilde{E}_{j}^{c, d, \alpha(y)}$ is given in (3.6).

We now show how to compute ${ }_{-1} D_{y}^{\alpha} L_{j}(y)$ and $\widetilde{D}_{-1}^{1, \alpha} L_{j}(y)$. To do this, we first expand $L_{j}(y)$ as

$$
L_{j}(y)=\sum_{i=0}^{N} l_{i}^{j} P_{i}^{c, d}(y)
$$

where

$$
l_{i}^{j}= \begin{cases}P_{i}^{c, d}\left(y_{j}\right) w_{j} / \gamma_{i}^{c, d}, & i=0, \ldots, N-1, \\ P_{N}^{c, d}\left(y_{j}\right) w_{j} /\left(\left(2+\frac{c+d+1}{N}\right) \gamma_{i}^{c, d}\right), & i=N,\end{cases}
$$

where $\left\{y_{j}, w_{j}\right\}, j=0, \ldots, N$ are the nodes and weights of the Jacobi-Gauss-Lobatto quadrature and $\gamma_{i}^{c, d}$ can be found in [30, Equation (3.109)]. Then, we have

$$
{ }_{-1} D_{y}^{\alpha} L_{j}(y)=\sum_{i=0}^{N} l_{i-1}^{j} D_{y}^{\alpha} P_{i}^{c, d}(y) .
$$

Hence, we only need to compute ${ }_{-1} D_{y}^{\alpha} P_{i}^{c, d}(y)$, which can be computed by using the three-term recurrence relation proposed in Theorem 3.4. The "red-block" entries in Fig. 3.1 are evaluated by using this formula.

As for $\widetilde{D}_{-1}^{1, \alpha} L_{j}(y)$, we apply the hybrid approach similar as in [5. In particular, when $y$ is close to 1 , we use the following formula

$$
\widetilde{D}_{-1}^{1, \alpha} L_{j}(y)=\sum_{i=0}^{N} l_{i}^{j} \widetilde{D}_{-1}^{1, \alpha} P_{i}^{c, d}(y),
$$

and $\widetilde{D}_{-1}^{1, \alpha} P_{i}^{c, d}(y), i=0, \ldots, N$ are computed by using the three-term recurrence relation proposed in Theorem 3.5. The "yellow-block" entries in Fig. 3.1 are evaluated by using this formula. When $y$ is far away from 1, we use the following Jacobi-Gauss-type quadrature

$$
\widetilde{D}_{-1}^{1, \alpha} L_{j}(y)=\frac{1}{\Gamma(-\alpha)} \sum_{i=0}^{N} \sum_{k=0}^{L} l_{i}^{j} \frac{P_{i}^{c, d}\left(\xi_{k, L}\right)}{\left(y-\xi_{k, L}\right)^{\alpha+1}} \omega_{k, L}^{c, d},
$$



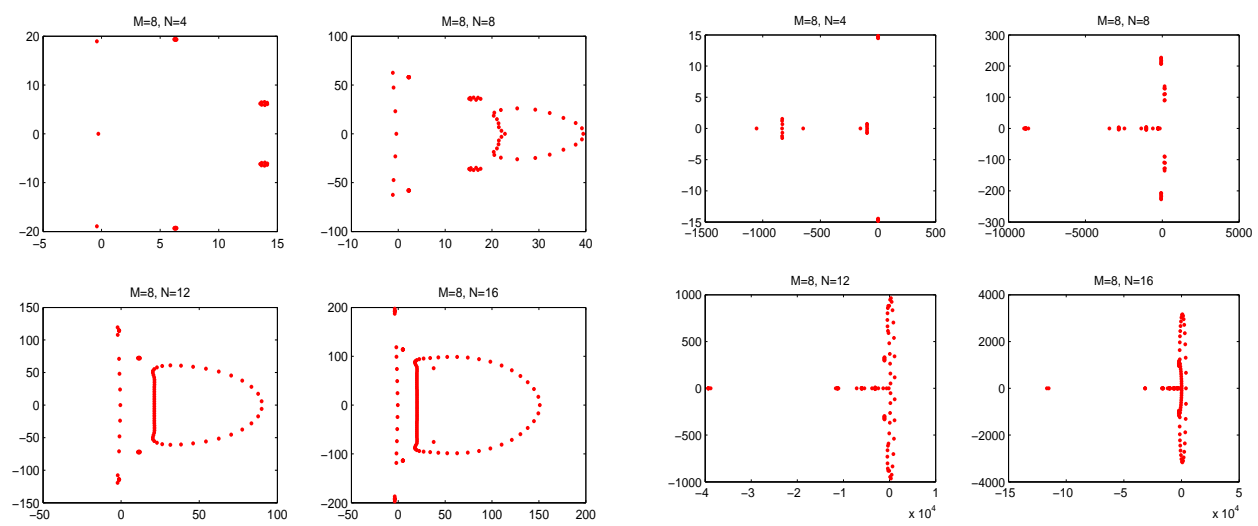

Fig. 3.2: Eigenvalues of MDFDM $\mathbf{D}^{\alpha}$ for $M=8$ and different values of $N$ with the uniform mesh. Left: $\alpha=1.01$, right: $\alpha=1.99$.
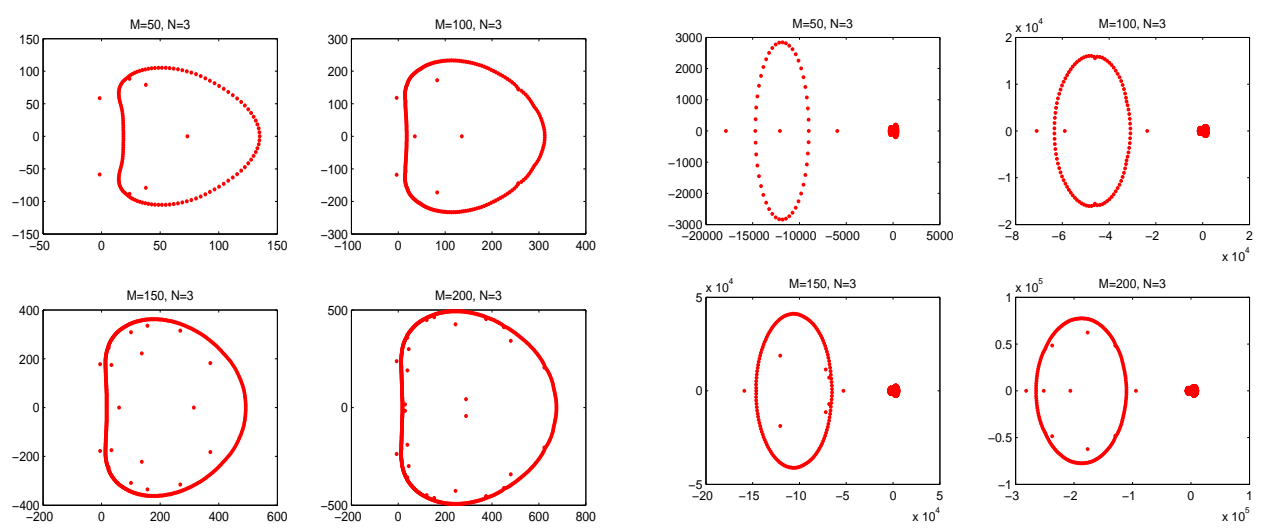

Fig. 3.3: Eigenvalues of MDFDM $\mathbf{D}^{\alpha}$ for $N=3$ and different values of $M$ with uniform mesh. Left: $\alpha=1.01$, right: $\alpha=1.99$.

where $\xi_{k, L}$ and $\omega_{k, L}$ are the Legendre-Gauss-type quadrature points and weights. The "green-block" entries in Fig. 3.1 are evaluated by using this formula.

3.3. Minimize the jump in the fluxes using a penalty method. Discontinuities of the (fractional or integer) fluxes of the nodal basis functions at the interfaces may lead to an unstable scheme when solving FDEs. Thus, we introduce a penalty parameter to stabilize the corresponding MDSCM by minimizing the jump in the integer fluxes.

To illustrate the possibility of the instability of the corresponding MDSCM, we show the distribution of eigenvalues of the MDFDM for different values of constantorder $\alpha=1.01,1.99$ with different $M, N$ using a uniform mesh. The other parameters are taken as $c=d=0, x_{L}=-1, x_{R}=1$. We observe from Figs. 3.2 3.3 that there exist eigenvalues whose real parts are positive, which would cause instability in the algorithm, for time-dependent problems.

Therefore, to overcome this issue, we minimize the jump in the integer fluxes 

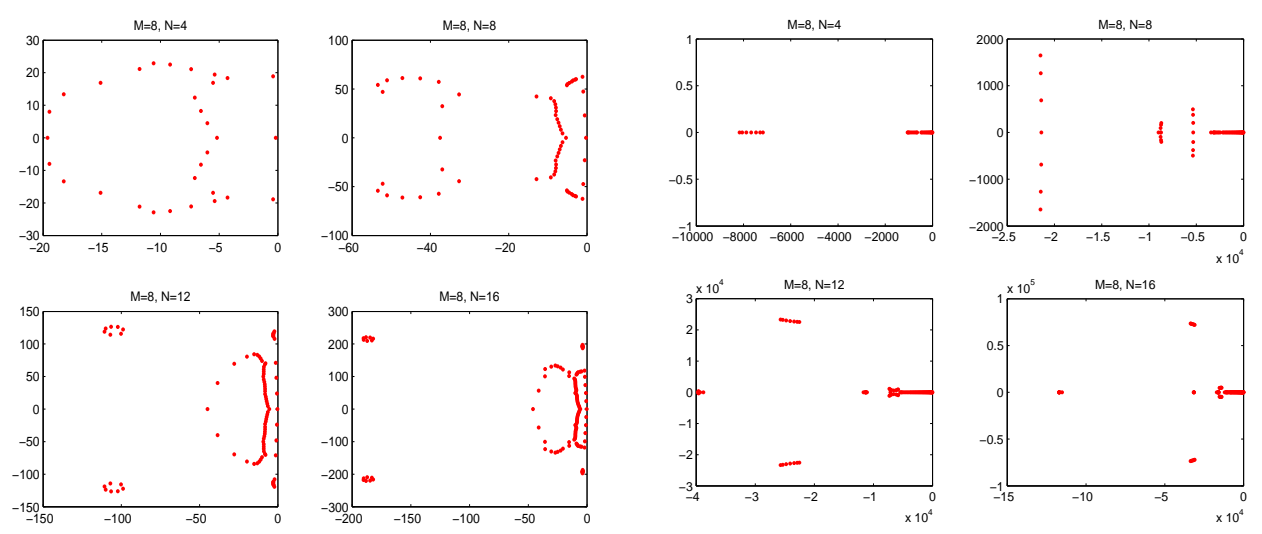

Fig. 3.4: Eigenvalues of MDFDM with penalty term, i.e., $\mathbf{D}^{\alpha}+\mathbf{R}$, for $M=8$ and different values of $N$ by using the uniform mesh. Left: $\alpha=1.01, \tau=1$, right: $\alpha=1.99, \tau=100$.

at the interfaces by using the penalty technique. In particular, by introducing the following penalty term

$$
R(x)=\tau\left[\frac{d u_{N}}{d x}\left(x^{+}\right)-\frac{d u_{N}}{d x}\left(x^{-}\right)\right], \quad x \in\left(x_{L}, x_{R}\right)
$$

where $\tau$ is a penalty parameter, we obtain a modified differentiation matrix $\mathbf{D}^{\alpha}+\mathbf{R}$, where $\mathbf{R}$ is given by

$$
\mathbf{R}=\left[\begin{array}{cccccc} 
& & & & \\
& & \mathbf{0} & & \\
& & & & \\
\mathbf{R}^{1} & \mathbf{R}^{2} & \cdots & \mathbf{R}^{M} & \widetilde{\mathbf{R}}
\end{array}\right]
$$

with

$$
\begin{aligned}
\mathbf{R}^{i} & =\tau\left(x_{m}\right)\left[\frac{d \psi_{n}^{i}\left(x_{m}^{+}\right)}{d x}-\frac{d \psi_{n}^{i}\left(x_{m}^{-}\right)}{d x}\right]_{m=1, \ldots, M-1 ; n=1, \ldots, N_{i}-1}, i=1, \ldots, M, \\
\widetilde{\mathbf{R}} & =\tau\left(x_{m}\right)\left[\frac{d \phi_{n}\left(x_{m}^{+}\right)}{d x}-\frac{d \phi_{n}\left(x_{m}^{-}\right)}{d x}\right]_{m, n=1 \ldots, M-1} \cdot
\end{aligned}
$$

It is noted that the $(M-1) \times(M-1)$ matix $\widetilde{\mathbf{R}}$ is tridiagonal, the $(M-1) \times\left(N_{1}-1\right)$ matrix $\mathbf{R}^{1}$ has only the first row with non-zero entries, the $(M-1) \times\left(N_{M}-1\right)$ matrix $\mathbf{R}^{M}$ has only the last row with non-zero entries, and the $(M-1) \times\left(N_{i}-1\right)$ matrix $\mathbf{R}^{i}, i=2, \ldots, M-1$, has only two, i.e., the $(i-1)$-th and $i$-th rows with non-zero entries. 

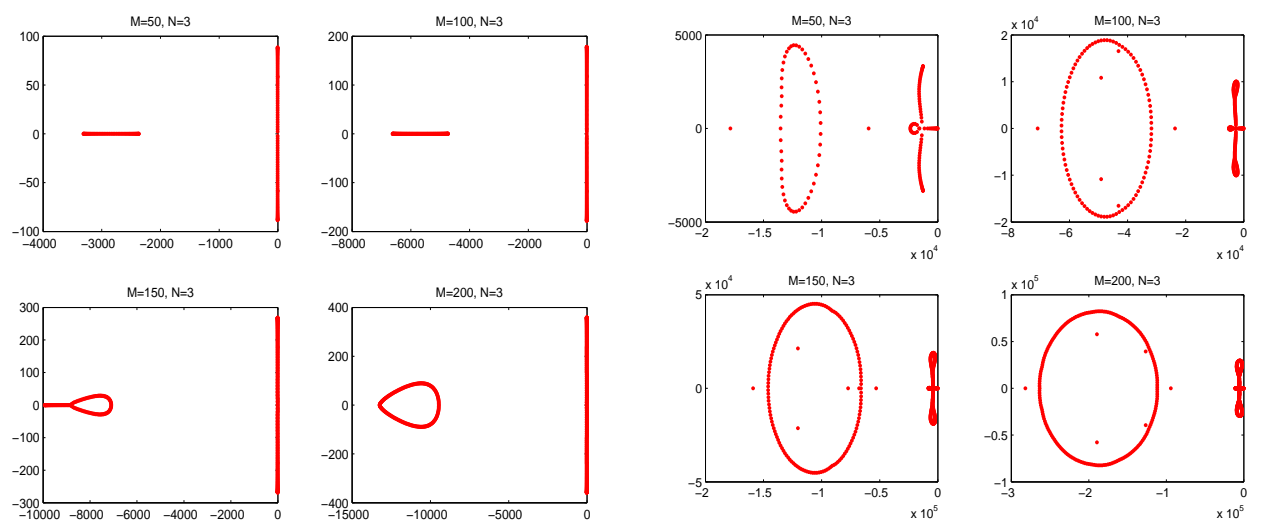

Fig. 3.5: Eigenvalues of MDFDM with penalty, i.e., $\mathbf{D}^{\alpha}+\mathbf{R}$, for $N=3$ and different values of $M$ by using the uniform mesh. Left: $\alpha=1.01, \tau=10$, right: $\alpha=1.99, \tau=$ 10.

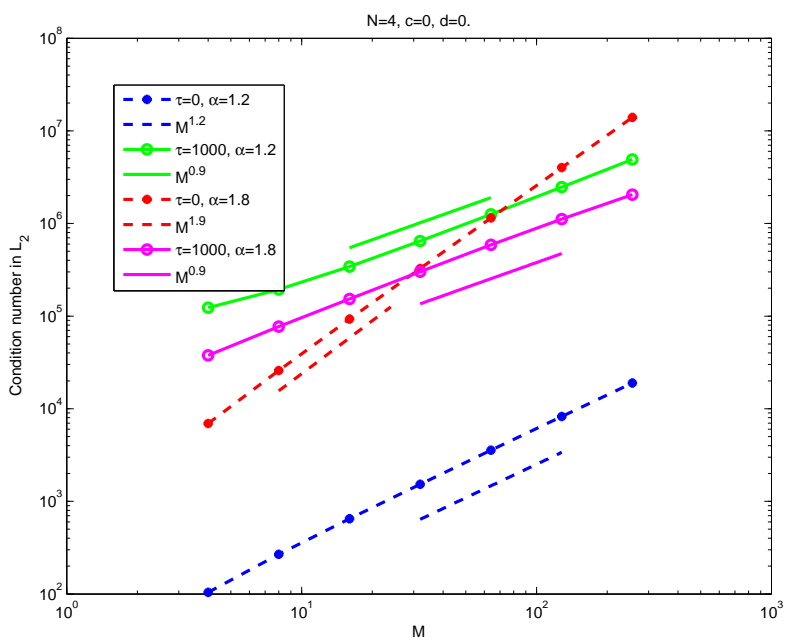

Fig. 3.6: Condition number with respect to $M$ with $(\tau=1000)$ or without $(\tau=0)$ penalty. The uniform mesh is used.

We show the distribution of the eigenvalues of the modified MDFDM, i.e., $\mathbf{D}^{\alpha}+\mathbf{R}$, in Figs. 3.4 3.5 with the same parameters used for the MDFDM. We observe that the real parts of all eigenvalues are negative in all cases by choosing suitable penalty parameters. The condition numbers are also investigated for some cases. In Fig. 3.6 we illustrate the condition numbers of the MDFDM in the $L_{2}$ norm. We see that the penalty term can slow down the growth of the condition number.

4. Application to the fractional Helmholtz equation and the fractional Burgers equation. We are now in the position of numerical tests. In the numerical tests, we use the following three types of mesh:

- Mesh 1: Uniform mesh: $x_{j}=x_{L}+\frac{\left(x_{R}-x_{L}\right) j}{M}, j=0, \ldots, M$. 
- Mesh 2: Graded mesh: $x_{j}=x_{L}+\left(x_{R}-x_{L}\right)\left(\frac{j}{M}\right)^{q}, \quad q>1, \quad j=0, \ldots, M$.

- Mesh 3: Geometric mesh: $x_{0}=x_{L}, x_{j}=x_{L}+\left(x_{R}-x_{L}\right) * q^{M-j}, \quad 0<q<$ $1, \quad j=1, \ldots, M$.

The number $\left\{N_{i}\right\}_{i=1}^{M}$ of collocation points in each element is the same for all three cases for simplification.

4.1. Fractional Helmholtz equation. Let $\Lambda:=\left(x_{L}, x_{R}\right)$ and $1<\alpha(x)<2$. In this subsection we apply the MDSCM to the following variable-order fractional Helmholtz equation

$$
\lambda^{2} u(x)-{ }_{x_{L}} D_{x}^{\alpha(x)} u(x)=f(x), \quad x \in \Lambda, \quad u\left(x_{L}\right)=u_{L}, u\left(x_{R}\right)=u_{R} .
$$

The stabilized MDSCM for (4.1) is to find $u_{N} \in \mathbb{V}_{N}$, such that

$$
\left[\lambda^{2} u_{N}-{ }_{x_{L}} D_{x}^{\alpha(x)} u_{N}-R(x)\right](x)=f(x), \forall x \in \mathbb{N}_{o} \backslash\left\{x_{L}, x_{R}\right\},
$$

and

$$
u_{N}\left(x_{L}\right)=u_{L}, \quad u_{N}\left(x_{R}\right)=u_{R} .
$$

The above two equations lead to the following linear system

$$
\left(\lambda^{2} \mathbb{I}-\mathbf{D}^{\alpha}-\mathbf{R}\right) \mathbf{u}=\mathbf{f}-\mathbf{r},
$$

where $\mathbb{I}$ is the unitary matrix and

$$
\begin{aligned}
\mathbf{x} & =\left[x_{1}^{1}, \cdots, x_{N_{1}-1}^{1}, x_{1}^{2}, \cdots, x_{N_{2}-1}^{2}, \cdots, x_{1}^{M}, \cdots, x_{N_{M}-1}^{M}, x_{1}, \cdots, x_{M-1}\right]^{T}, \\
\mathbf{f} & =f(\mathbf{x})-u_{L}\left(\lambda^{2} \phi_{0}(\mathbf{x})-{ }_{x_{L}} D_{x}^{\alpha(x)} \phi_{0}(\mathbf{x})\right)-u_{R}\left(\lambda^{2} \phi_{M}(\mathbf{x})-{ }_{x_{L}} D_{x}^{\alpha(x)} \phi_{M}(\mathbf{x})\right), \\
\mathbf{r} & =\tau(\mathbf{x})\left\{u_{L}\left[\frac{d \phi_{0}\left(\mathbf{x}^{-}\right)}{d x}-\frac{d \phi_{0}\left(\mathbf{x}^{+}\right)}{d x}\right]+u_{R}\left[\frac{d \phi_{M}\left(\mathbf{x}^{-}\right)}{d x}-\frac{d \phi_{M}\left(\mathbf{x}^{+}\right)}{d x}\right]\right\} .
\end{aligned}
$$

and $\mathbf{u}=u_{N}(\mathbf{x})$.

EXAMPLE 4.1. The first test of MDSCM is to consider the problem 4.1) with $\left[x_{L}, x_{R}\right]=[-1,1]$. The exact solution is taken as $u(x)=\sin (\pi x)$, so the homogeneous boundary conditions are implemented. The term ${ }_{x_{L}} D_{x}^{\alpha(x)} u(x)$ is approximated by

$$
{ }_{-1} D_{x}^{\alpha(x)} \sin (\pi x) \approx \sum_{k=0}^{L}(-1)^{k+1} \frac{\pi^{2 k+1}(x+1)^{2 k+1-\alpha(x)}}{\Gamma(2 k+2-\alpha(x))},
$$

with $L=50$ to compute the right hand function (RHF) $f(x)$. For $\alpha(x)$, we consider the following two cases:

1. The constant-order $\alpha=1.1,1.5,1.9$.

2. The variable-order $\alpha(x)=1.1+\frac{x+1}{2.5}$.

The aim of this example is to test the accuracy of the proposed method for the smooth solution. In this example the uniform mesh is used. The maximum errors between the numerical solution and exact solution are shown in Figs. 4.14.2, We observe from the left plots of Fig. 4.1 and Fig. 4.2 that the spectral accuracy is obtained for both constant-order and variable-order fractional derivative. Also, the good accuracy can be obtained by $h$-refinement (right plots of Fig. 4.1 and Fig. 4.2). We also observe from Figs. 4.1, 4.2 that the accuracy can be improved significantly by the penalty method. 

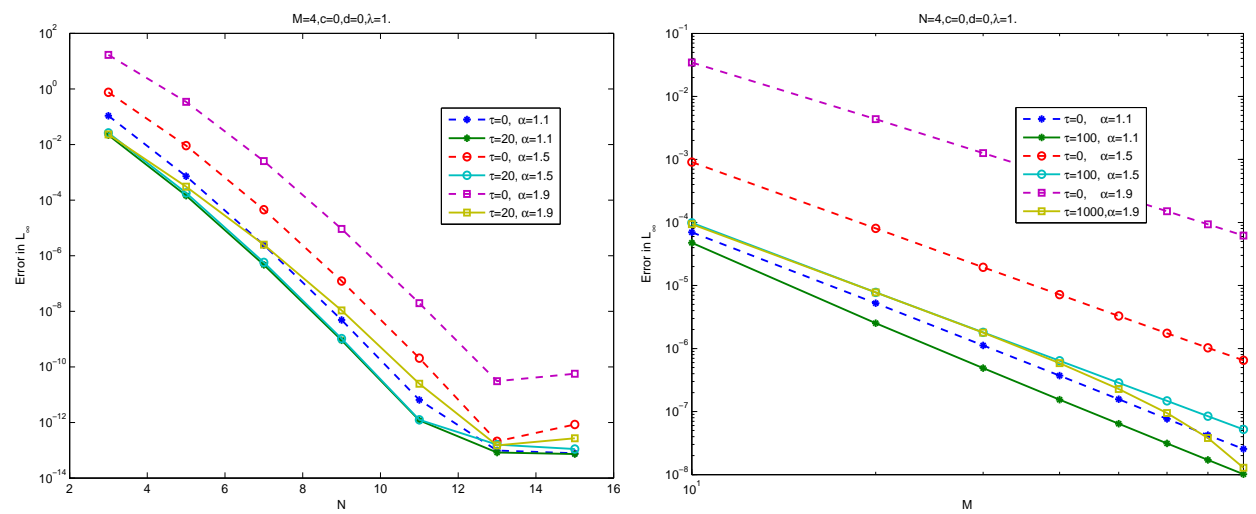

Fig. 4.1: $L_{\infty}$-error for Example 4.1 with the uniform mesh (Mesh 1). Left: $p$ refinement $(M=4)$, right: $h$-refinement $(N=4)$.
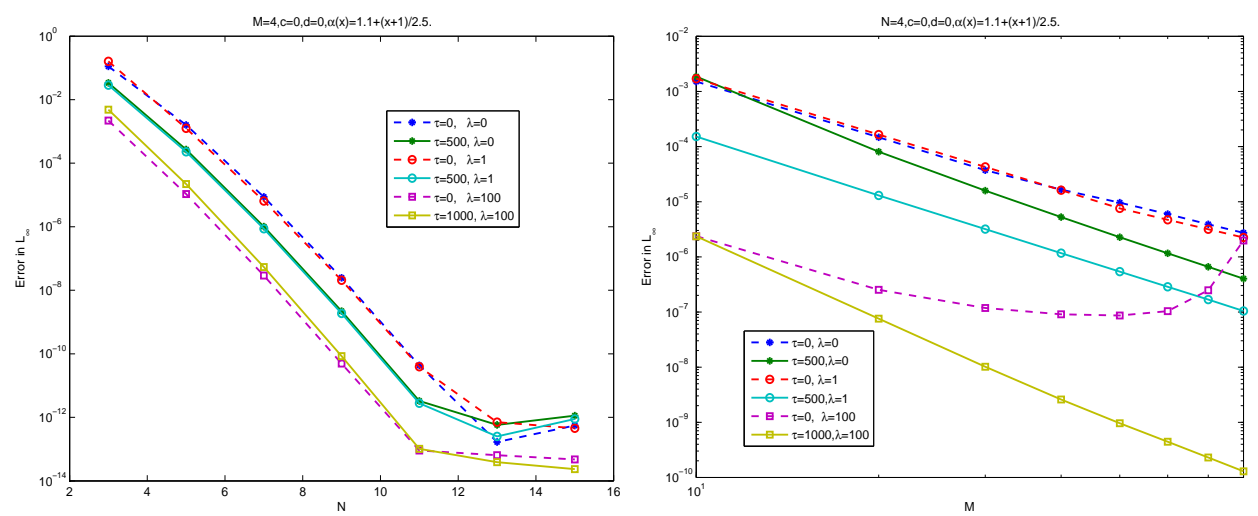

Fig. 4.2: Error in $L_{\infty}$ for Example 4.1 with the uniform mesh (Mesh 1). Left: $p$ refinement $(M=4)$, right: $h$-refinement $(N=4)$.

EXAMPLE 4.2. In the second test of the MDSCM we also consider the model problem (4.1) with $\left[x_{L}, x_{R}\right]=[-1,1]$. The exact solution is taken as $u(x)=(1-$ $x)(1+x)^{\alpha(x)-1}$, and then we can obtain the RHF

$$
f(x)=\lambda^{2} u(x)+\Gamma(1+\alpha(x)) .
$$

When $\lambda=0$, we obtain a smooth RHF $f$ if the order $\alpha(x)$ is smooth. We can see that the exact solution has very low regularity since $0<\alpha(x)-1<1$. We aim to find out whether the MDSCM can give a good approximation for a low regularity solution.

The maximum errors between the numerical and exact solutions are plotted in Figs. 4.3 4.5. We see that the MDSCM without penalty $(\tau=0)$ hardly achieves any accuracy when $\alpha(x)-1 \in(0,1)$, whereas the MDSCM with the penalty term $(\tau \neq 0)$ always converges. Also, we can observe that the geometric mesh (Mesh 3 ) can achieve better accuracy than the graded mesh (Mesh 2) when $\alpha(x)-1$ is close to zero. We also compare with the spectral element method ("SEM") proposed in 23. and we see 

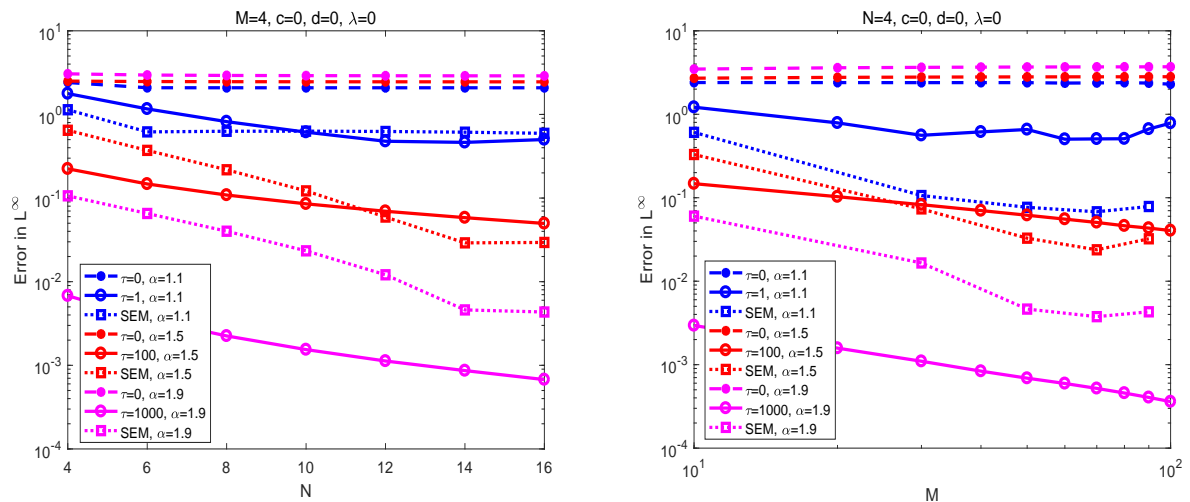

Fig. 4.3: Error in $L_{\infty}$ for Example 4.2 with the uniform mesh (Mesh 1). Left: $p$ refinement $(M=4)$, right: $h$-refinement $(N=4)$.
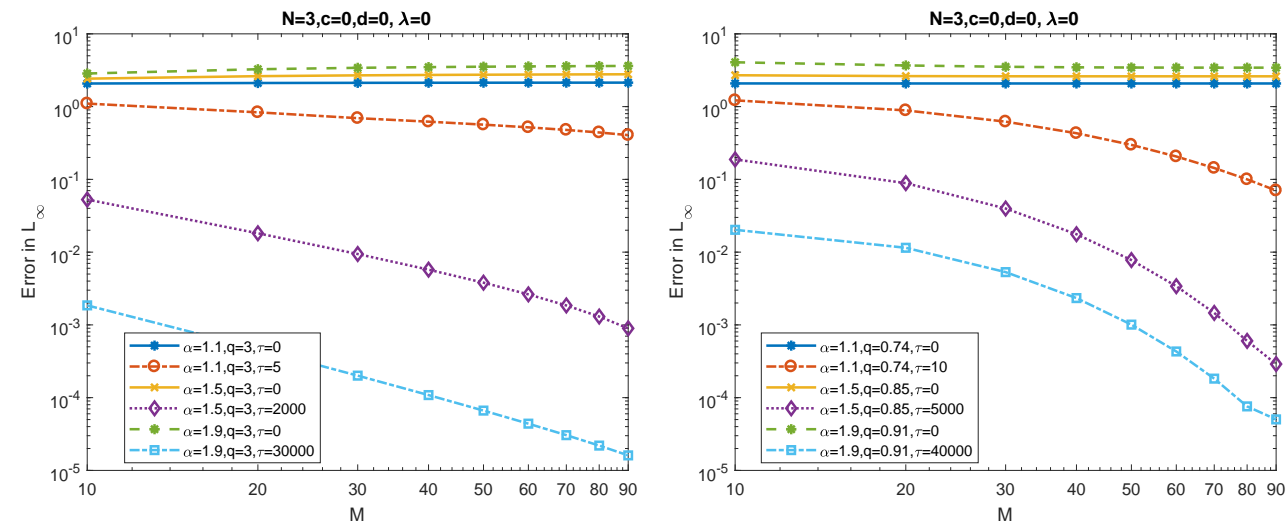

Fig. 4.4: Error in $L_{\infty}$ for Example 4.2 for different values of $\alpha$. Left: graded mesh, right: geometric mesh.

that while it is better than the MDSCM, the penalty-based version exhibits superior performance overall.

4.2. Fractional Burgers equation. In this subsection we employ the MDSCM to solve a time dependent problem, i.e., the following fractional Burgers equation (FBE)

$$
\partial_{t} u(x, t)+u(x, t) \partial_{x} u(x, t)=\epsilon D^{\alpha(x, t)} u(x, t),
$$

subject to homogeneous Dirichlet boundary conditions and initial condition $u(x, 0)=$ $u_{0}(x)$, where $\epsilon>0,1<\alpha(x, t)<2,(x, t) \in(-1,1) \times(0,1]$.

For the time discretization, we employ a semi-implicit time-discretization scheme, namely, the two-step second-order Crank-Nicolson/leapfrog scheme, then, the full 

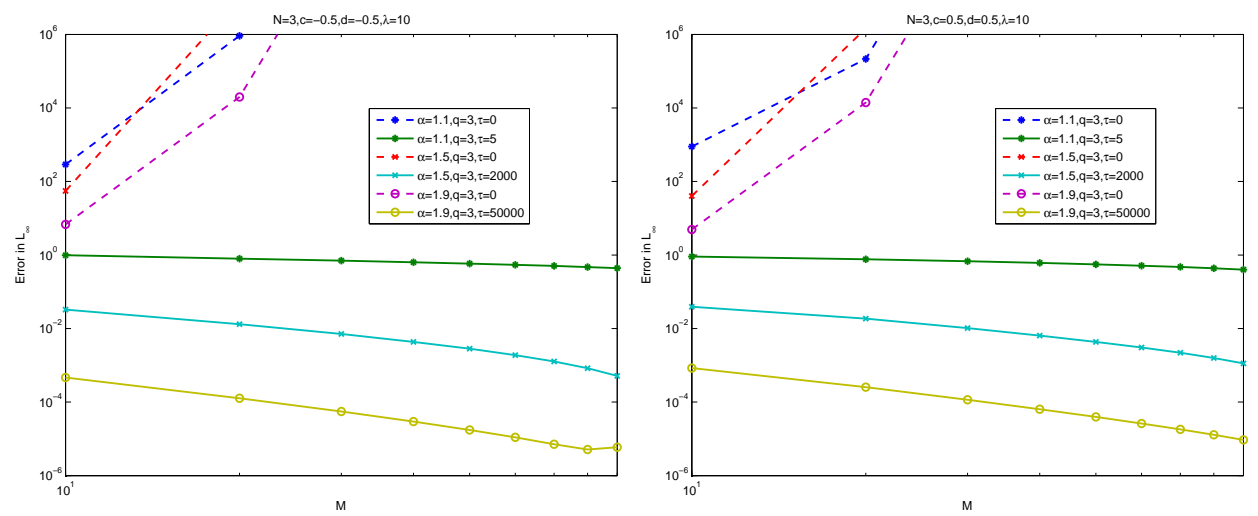

Fig. 4.5: Error in $L_{\infty}$ for Example 4.2 with graded mesh and different Jacobi interpolants. Left: $c=d=-1 / 2$, right: $c=d=1 / 2$.

discretization scheme reads as: for $n=1,2, \ldots$,

$$
\left\{\begin{array}{l}
\left(\mathbb{I}-\Delta t \epsilon\left(\mathbf{D}^{\alpha^{n+1}}+\mathbf{R}\right)\right) \mathbf{u}^{n+1}=\mathbf{g} \\
\mathbf{u}^{1}=\mathbf{u}^{0}+\Delta t \epsilon \mathbf{D}^{\alpha^{0}} \mathbf{u}^{0}-\Delta t\left(\operatorname{diag}\left(\mathbf{u}^{0}\right) \mathbf{D} \mathbf{u}^{0}\right) \\
\mathbf{u}^{0}=u_{0}(\mathbf{x})
\end{array}\right.
$$

where

$$
\mathbf{g}=\left(\mathbb{I}+\Delta t \epsilon \mathbf{D}^{\alpha^{n-1}}\right) \mathbf{u}^{n-1}-2 \Delta t\left(\operatorname{diag}\left(\mathbf{u}^{n}\right) \mathbf{D} \mathbf{u}^{n}\right),
$$

$\mathbf{D}^{\alpha^{n}}, \mathbf{R}$ and $\mathbf{D}$ are the MDFDM of $\alpha$-order, the penalty matrix and the first-order differentiation matrix, respectively.

EXAMPLE 4.3. In this example, we consider the initial condition $u_{0}(x)=\sin (\pi x)$ and the following five cases of fractional order considered in [45]:

- Case 1: (constant-order) $\alpha(x, t)=1.1,1.2,1.3,1.5,1.8$;

- Case 2: (monotonic increasing-order) $\alpha(x, t)=1+\frac{5+4 x}{10}$;

- Case 3: (monotonic decreasing-order) $\alpha(x, t)=1+\frac{5-4 x}{10}$;

- Case 4: (nonsmooth order) $\alpha(x, t)=\frac{4}{5}|\sin (10 \pi(x-t))|+1.1$;

- Case 5: (nonsmooth order) $\alpha(x, t)=\frac{4|x t|}{5}+1.1$.

We first consider the constant-order case, i.e., Case 1. We show the numerical solutions of the FBE (4.5) at time $t=1$ in Fig. 4.6 for different values of $\alpha$ by using the uniform mesh. A comparison of the numerical solutions for $\alpha=1.5$ is also shown in Fig. 4.7 by using $h$ or $p$ refinement. Observe that the obtained numerical result is the same as the one obtained in [45, Fig. 5] and the solutions near the left boundary have sharp transitions, especially for smaller values of $\alpha$.

Furthermore, to illustrate the effect of the proposed MDSCM in resolving the issue of singularities, we show the numerical solutions for $\alpha=1.1$ at time $t=1$ in Fig. 4.8 with uniform mesh (left plot) and graded mesh or geometric mesh (right plot), respectively. The reference solution is computed by using the graded mesh with $M=200, q=3$. We observe that the one-domain spectral method, although it has better accuracy at the left boundary, it exhibits oscillations, which propagate and eventually renderer the solution erroneous. However, the $h$-refinement can resolve this 


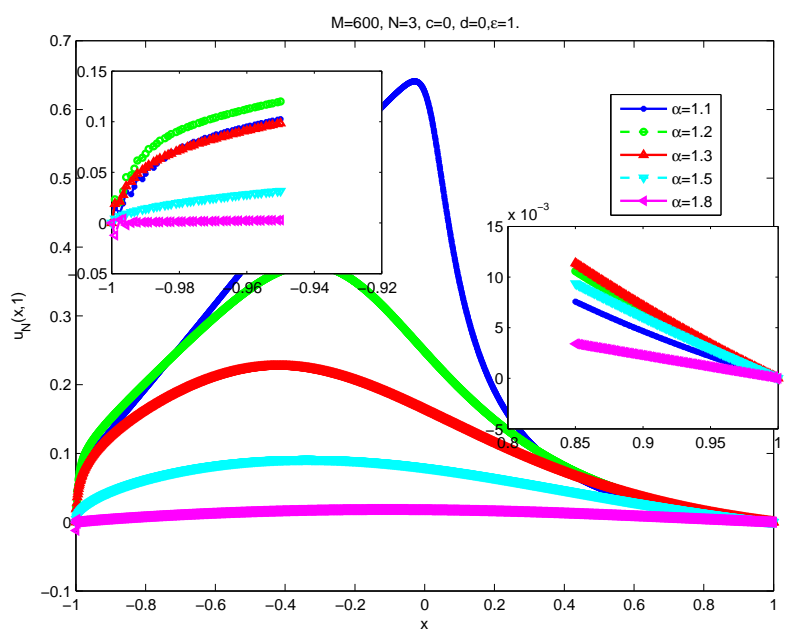

Fig. 4.6: Numerical solutions at $t=1$ for Example 4.3 (Case 1) with the uniform mesh (Mesh 1). $\epsilon=1, M=600, N=3, c=d=0, \tau=10^{3}, \Delta t=10^{-3}$.
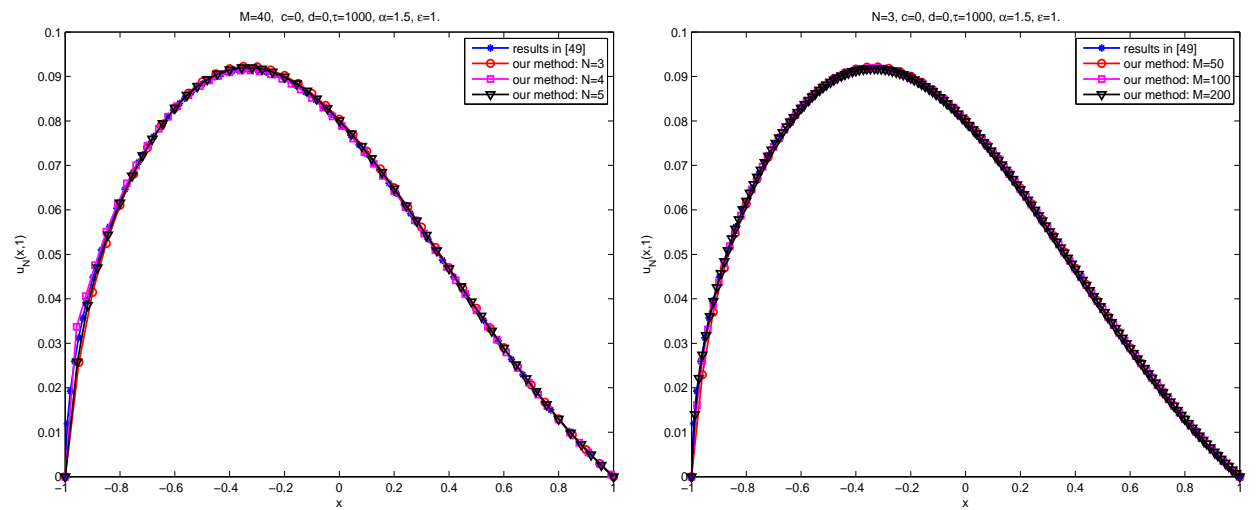

Fig. 4.7: Comparison of numerical solutions at $t=1$ for Example 4.3 with the one obtained in [49] for $\alpha=1.5$. Left: $p$-refinement, right: $h$-refinement.

issue. Moreover, by using graded or geometric meshes, we can further enhance the accuracy of the solution. Also, the result by using the graded mesh is more accurate compared with the results obtained by using the uniform or the geometric mesh. We point out here that for the geometric mesh, we first divide the interval $[-1,1]$ into two subintervals $[-1,-0.95]$ and $[-0.95,1]$, and subsequently use a geometric mesh for the first subinterval with 10 spectral elements and $q=0.5$ while we use a uniform mesh for the second subinterval with $M-10$ elements, where $M$ is the total number of elements over the entire interval.

We also study the behavior of the solutions with different values of viscosity $\varepsilon$. The numerical results for different values of fractional order $\alpha=1.3,1.8$ are shown in Fig. 4.9. We observe high degree of sharpness when the value of viscosity $\varepsilon$ is very small, and this sharpness can be captured by using the proposed MDSCM. 

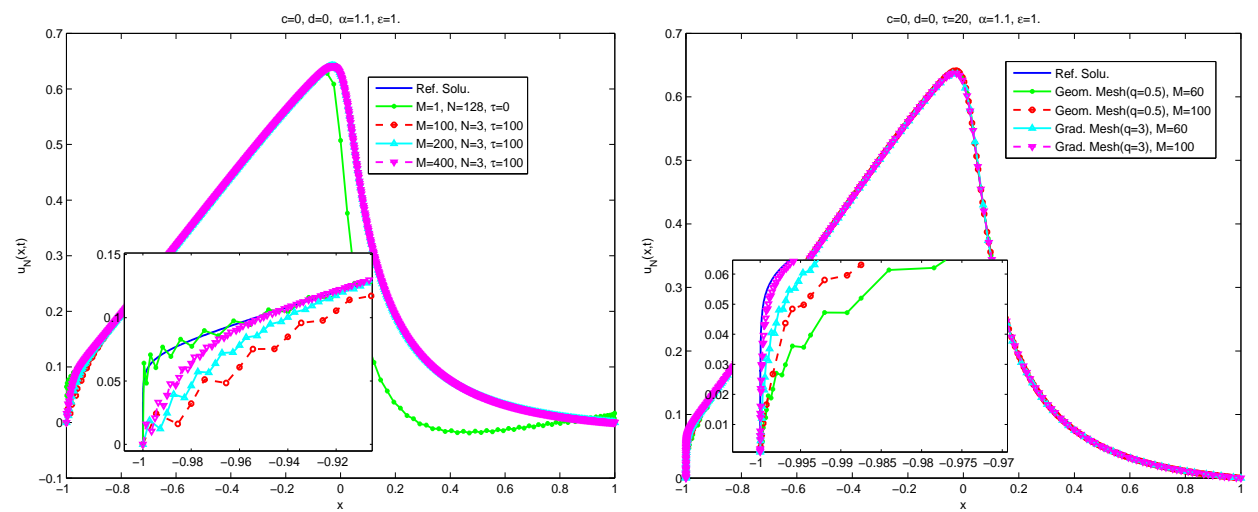

Fig. 4.8: Numerical solutions at $t=1$ for Example 4.3 (Case 1) and the value of the fractional order $\alpha=1.1$. Left: uniform mesh, right: graded mesh and geometric mesh.
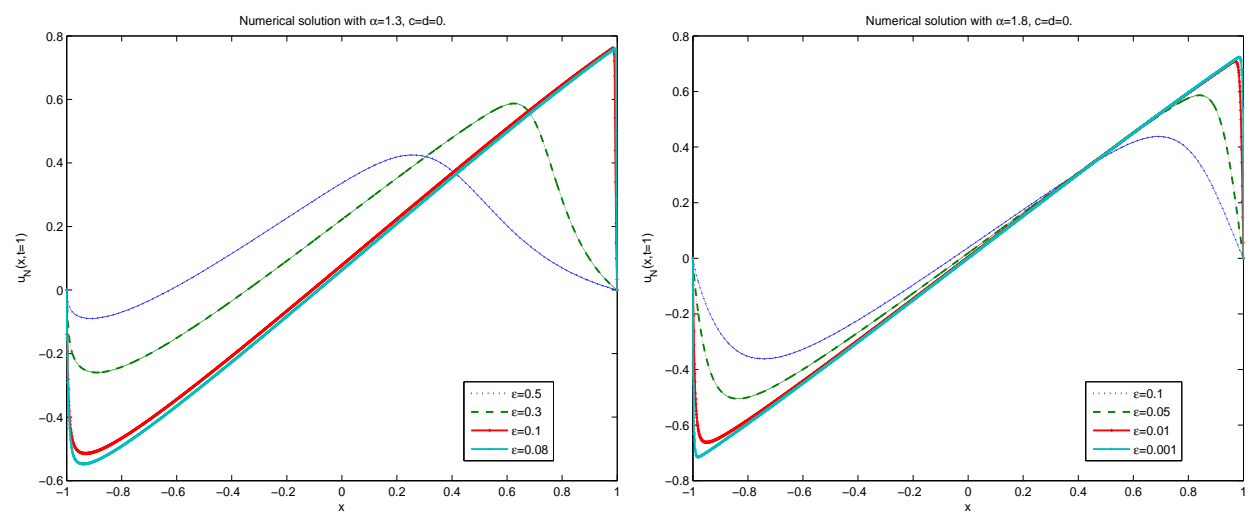

Fig. 4.9: Numerical solutions at $t=1$ for Example 4.3 with the uniform mesh (Mesh 1) and different values of viscosity $\varepsilon . M=600, N=3, c=d=0, \tau=10^{4}, \Delta t=10^{-3}$. Left: $\alpha=1.3$, right: $\alpha=1.8$.

We now consider the variable-order cases, i.e., Cases 2-5. The numerical solutions at time $t=1$ are shown in Fig. 4.10. Again, we see that the oscillations can be eliminated by refining the mesh, see, e.g, upper left and lower left plots of Fig. 4.10,

5. Conclusion. In this paper, we present a multi-domain spectral collocation method (MDSCM) for numerically solving fractional partial differential equations that cannot be easily solved with Galerkin single- or multi-domain spectral methods. We construct a set of nodal basis functions and derive the variable-order multi-domain fractional differentiation matrix, which can be computed efficiently and are used to formulate the proposed method. We also employ a penalty technique by minimizing the jump in (integer) fluxes to stabilize the MDSCM, which can slow down the growth of the condition number of the corresponding differentiation matrix.

Various numerical tests show that the MDSCM achieves spectral accuracy with 

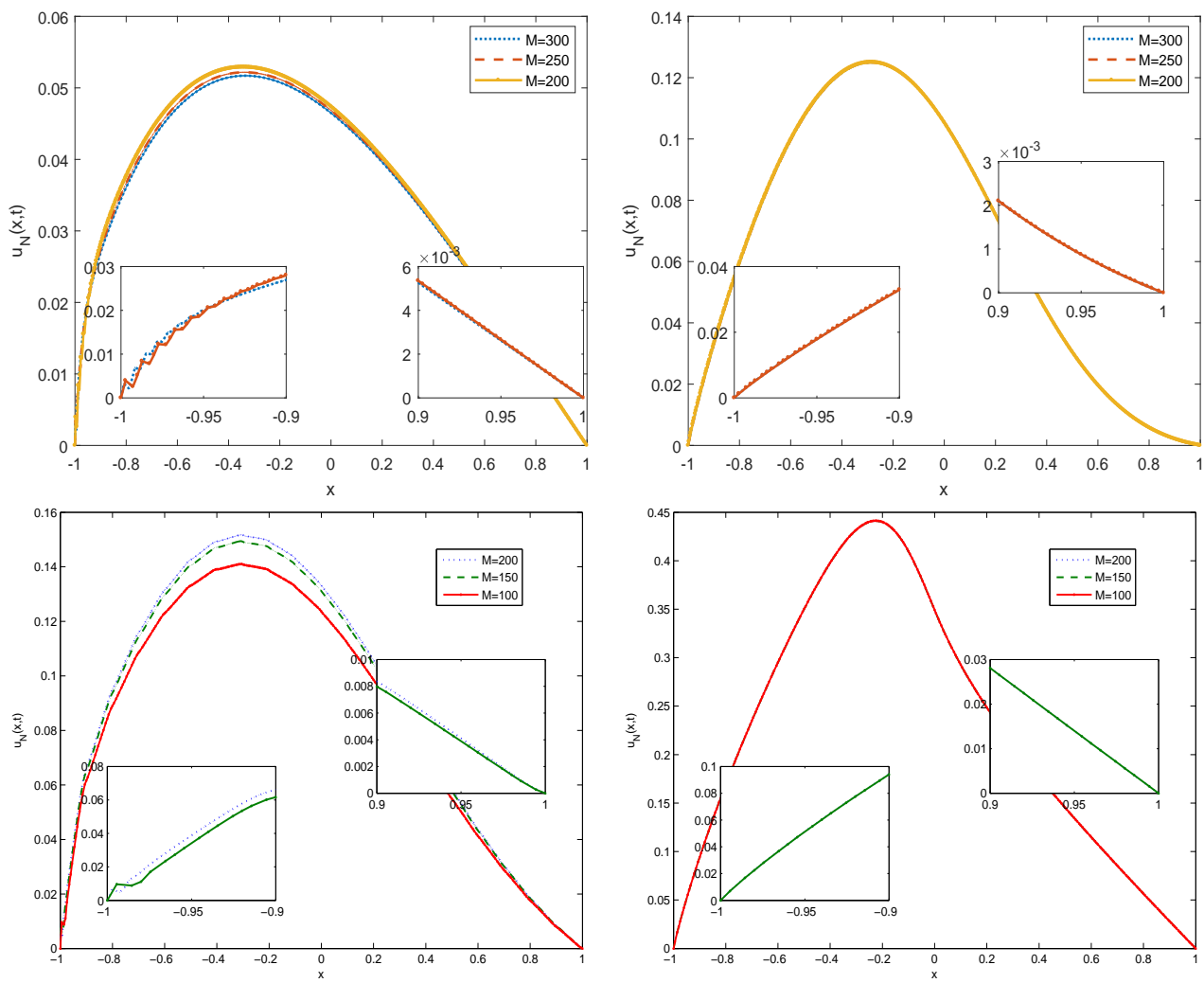

Fig. 4.10: Numerical solutions at $t=1$ for Example 4.3 with the uniform mesh (Mesh 1) and $\varepsilon=1, N=3, c=d=0, \tau=10^{5}, \Delta t=10^{-3}$. Upper left: Case 2: $\alpha=\frac{5+4 x}{10}+1$, upper right: Case 3: $\alpha=\frac{5-4 x}{10}+1$, lower left: Case 4: $\alpha=$ $\frac{4|\sin (10 \pi(x-t))|}{5}+1.1$, lower right: Case $5: \alpha=\frac{4}{5}|x t|+1.1$.

respect to the order of polynomial under the assumption that the exact solution is sufficiently smooth. We also demonstrate that the MDSCM has a big advantage in obtaining high accuracy when the solutions have low regularity compared with the single-domain spectral method. Also, the multi-domain method is more flexible in performing $h$ and $p$ refinement for fractional boundary value problems as well as problems with interior regions of low regularity or very steep gradients. In addition, it is easy to apply MDSCM to variable-coefficient problems with variable-order fractional derivatives. Moreover, by using the penalty method, we don't only stabilize the MDSCM but also improve greatly the accuracy of the scheme. It is especially effective for solutions with very low regularity, in which case, the non-penalized MDSCM fails to converge while the penalized MDSCM does converge in the $L^{\infty}$ sense. Furthermore, by choosing a suitable penalty parameter, the penalty-based MDSCM exhibits superior performance compared with the non-penalty version and the spectral element method based on the Galerkin formulation. Unfortunately, currently we do not have a rigorous theory to show how to choose the optimal value of the penalty parameter, which is an issue of great practical interest, and this should be addressed in future work. Another open issue is the optimal penalty procedure. In the present 
work, we penalize the integer fluxes, but another possibility is to penalize the fractional fluxes; however, in this case, we need to use the non-polynomial basis, namely, the poly-fractonomials proposed in 39 .

\section{REFERENCES}

[1] R. L. BAgley And P. Torvik, A theoretical basis for the application of fractional calculus to viscoelasticity, Journal of Rheology, 27 (1983), pp. 201-210.

[2] R. L. Bagley and P. J. TORVIK, Fractional calculus in the transient analysis of viscoelastically damped structures, AIAA journal, 23 (1985), pp. 918-925.

[3] R. T. BAILLIE, Long memory processes and fractional integration in econometrics, J. Econometrics, 73 (1996), pp. 5-59.

[4] E. BARKAI, Fractional fokker-planck equation, solution, and application, Physical Review E, 63 (2001), p. 046118.

[5] F. Chen, Q. Xu, and J. S. Hesthaven, A multi-domain spectral method for time-fractional differential equations, J. Comput. Phys., 293 (2015), pp. 157-172.

[6] S. Chen, J. Shen, And L.-L. Wang, Generalized Jacobi functions and their applications to fractional differential equations, Math. Comp., 85 (2016), pp. 1603-1638.

[7] W. Deng, Finite element method for the space and time fractional Fokker-Planck equation, SIAM J. Numer. Anal., 47 (2008), pp. 204-226.

[8] K. Diethelm AND A. D. Freed, On the solution of nonlinear fractional-order differential equations used in the modeling of viscoplasticity, in Scientific Computing in Chemical Engineering II, Springer, 1999, pp. 217-224.

[9] N. J. Ford, J. XiaO, AND Y. Yan, A finite element method for time fractional partial differential equations, Fract. Calc. Appl. Anal., 14 (2011), pp. 454-474.

[10] Y. JIANG AND J. MA, High-order finite element methods for time-fractional partial differential equations, J. Comput. Appl. Math., 235 (2011), pp. 3285-3290.

[11] E. Kharazmi, M. Zayernouri, and G. E. Karniadakis, A Petrov-Galerkin spectral element method for fractional elliptic problems, Comput. Methods Appl. Mech. Engrg., 324 (2017), pp. 512-536.

[12] K. Kikuchi And A. Negoro, On Markov process generated by pseudodifferential operator of variable order, Osaka J. Math., 34 (1997), pp. 319-335.

[13] C. Li, F. Zeng, And F. Liu, Spectral approximations to the fractional integral and derivative, Fract. Calc. Appl. Anal., 15 (2012), pp. 383-406.

[14] X. Li And C. Xu, A space-time spectral method for the time fractional diffusion equation, SIAM J. Numer. Anal., 47 (2009), pp. 2108-2131.

[15] X. Li AND C. XU, Existence and uniqueness of the weak solution of the space-time fractional diffusion equation and a spectral method approximation, Commun. Comput. Phys., 8 (2010), pp. 1016-1051.

[16] Y. Lian, Y. Ying, S. Tang, S. Lin, G. J. Wagner, and W. K. Liu, A Petrov-Galerkin finite element method for the fractional advection-diffusion equation, Comput. Methods Appl. Mech. Engrg., 309 (2016), pp. 388-410.

[17] Y. Lin AND C. XU, Finite difference/spectral approximations for the time-fractional diffusion equation, J. Comput. Phys., 225 (2007), pp. 1533-1552.

[18] F. LiU, V. ANh, AND I. TuRner, Numerical solution of the space fractional Fokker-Planck equation, in Proceedings of the International Conference on Boundary and Interior LayersComputational and Asymptotic Methods (BAIL 2002), vol. 166, 2004, pp. 209-219.

[19] F. MAINARDI, Fractional calculus: some basic problems in continuum and statistical mechanics, in Fractals and fractional calculus in continuum mechanics (Udine, 1996), vol. 378 of CISM Courses and Lect., Springer, Vienna, 1997, pp. 291-348.

[20] Z. Mao, S. Chen, And J. Shen, Efficient and accurate spectral method using generalized Jacobi functions for solving Riesz fractional differential equations, Appl. Numer. Math., 106 (2016), pp. 165-181.

[21] Z. MaO AND G. E. KaRniadakis, A spectral method (of exponential convergence) for singular solutions of the diffusion equation with general two-sided fractional derivative, SIAM J. Numer. Anal., 56 (2018), pp. 24-49.

[22] Z. Mao AND J. Shen, Efficient spectral-Galerkin methods for fractional partial differential equations with variable coefficients, J. Comput. Phys., 307 (2016), pp. 243-261.

[23] Z. Mao And J. Shen, Spectral element method with geometric mesh for two-sided fractional differential equations, Adv. Comput. Math., 44 (2018), pp. 745-771.

[24] M. M. Meerschaert, H.-P. Scheffler, and C. Tadjeran, Finite difference methods for two- 
dimensional fractional dispersion equation, J. Comput. Phys., 211 (2006), pp. 249-261.

[25] M. M. MeERschaert AND C. TADJERAn, Finite difference approximations for fractional advection-dispersion flow equations, J. Comput. Appl. Math., 172 (2004), pp. 65-77.

[26] R. Metzler and J. Klafter, The random walk's guide to anomalous diffusion: a fractional dynamics approach, Phys. Rep., 339 (2000), p. 77.

[27] R. Metzlen AND J. Klafter, The restaurant at the end of the random walk: recent developments in the description of anomalous transport by fractional dynamics, J. Phys. A, 37 (2004), pp. R161-R208.

[28] Y. A. Rossikhin And M. V. Shitikova, Applications of fractional calculus to dynamic problems of linear and nonlinear hereditary mechanics of solids, Applied Mechanics Reviews, 50 (1997), pp. 15-67.

[29] M. D. Ruiz-Medina, V. V. Anh, And J. M. Angulo, Fractional generalized random fields of variable order, Stochastic Anal. Appl., 22 (2004), pp. 775-799.

[30] J. Shen, T. TANG, AND L.-L. WANG, Spectral methods: algorithms, analysis and applications, vol. 41, Springer Science \& Business Media, 2011.

[31] Z.-Z. Sun AND X. Wu, A fully discrete difference scheme for a diffusion-wave system, Appl. Numer. Math., 56 (2006), pp. 193-209.

[32] C. Tadjeran and M. M. Meerschaert, A second-order accurate numerical method for the two-dimensional fractional diffusion equation, J. Comput. Phys., 220 (2007), pp. 813-823.

[33] C. Tadjeran, M. M. Meerschaert, and H.-P. Scheffler, A second-order accurate numerical approximation for the fractional diffusion equation, J. Comput. Phys., 213 (2006), pp. 205213.

[34] W. Y. Tian, W. Deng, And Y. Wu, Polynomial spectral collocation method for space fractional advection-diffusion equation, Numer. Methods Partial Differential Equations, 30 (2014), pp. 514-535.

[35] H. WANG And T. S. BASU, A fast finite difference method for two-dimensional space-fractional diffusion equations, SIAM J. Sci. Comput., 34 (2012), pp. A2444-A2458.

[36] H. WANG AND K. WANG, An $O\left(N \log ^{2} N\right)$ alternating-direction finite difference method for two-dimensional fractional diffusion equations, J. Comput. Phys., 230 (2011), pp. 78307839 .

[37] H. WAng, D. YAng, And S. Zhu, A Petrov-Galerkin finite element method for variablecoefficient fractional diffusion equations, Comput. Methods Appl. Mech. Engrg., 290 (2015), pp. 45-56.

[38] Q. Xu And J. S. Hesthaven, Stable multi-domain spectral penalty methods for fractional partial differential equations, J. Comput. Phys., 257 (2014), pp. 241-258.

[39] M. Zayernouri and G. E. Karniadakis, Fractional Sturm-Liouville eigen-problems: theory and numerical approximation, J. Comput. Phys., 252 (2013), pp. 495-517.

[40] M. Zayernouri and G. E. Karniadakis, Discontinuous spectral element methods for timeand space-fractional advection equations, SIAM J. Sci. Comput., 36 (2014), pp. B684-B707.

[41] M. ZaYernouri AND G. E. KARNIADAKIS, Exponentially accurate spectral and spectral element methods for fractional ODEs, J. Comput. Phys., 257 (2014), pp. 460-480.

[42] M. Zayernouri and G. E. Karniadakis, Fractional spectral collocation method, SIAM J. Sci. Comput., 36 (2014), pp. A40-A62.

[43] F. Zeng, F. Liu, C. Li, K. Burrage, I. Turner, and V. Anh, A Crank-Nicolson ADI spectral method for a two-dimensional Riesz space fractional nonlinear reaction-diffusion equation, SIAM J. Numer. Anal., 52 (2014), pp. 2599-2622.

[44] F. Zeng, Z. MaO, ANd G. E. KarniadAKIS, A generalized spectral collocation method with tunable accuracy for fractional differential equations with end-point singularities, SIAM J. Sci. Comput., 39 (2017), pp. A360-A383.

[45] F. Zeng, Z. Zhang, And G. E. Karniadakis, A generalized spectral collocation method with tunable accuracy for variable-order fractional differential equations, SIAM J. Sci. Comput., 37 (2015), pp. A2710-A2732.

[46] Y.-N. Zhang, Z.-Z. Sun, AND H.-W. Wu, Error estimates of Crank-Nicolson-type difference schemes for the subdiffusion equation, SIAM J. Numer. Anal., 49 (2011), pp. 2302-2322.

[47] X. Zhao, Z.-Z. Sun, AND Z.-P. HAO, A fourth-order compact ADI scheme for two-dimensional nonlinear space fractional Schrödinger equation, SIAM J. Sci. Comput., 36 (2014), pp. A2865-A2886.

[48] Y. Zheng, C. Li, And Z. Zhao, A note on the finite element method for the space-fractional advection diffusion equation, Comput. Math. Appl., 59 (2010), pp. 1718-1726.

[49] H. Zhou, W. Tian, AND W. Deng, Quasi-compact finite difference schemes for space fractional diffusion equations, J. Sci. Comput., 56 (2013), pp. 45-66.

[50] P. Zhuang, F. Liu, V. Anh, And I. Turner, Numerical methods for the variable-order frac- 
tional advection-diffusion equation with a nonlinear source term, SIAM J. Numer. Anal., 47 (2009), pp. 1760-1781. 